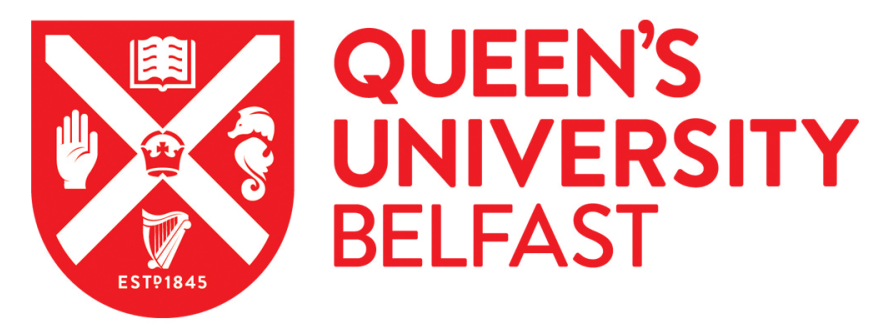

\title{
Rational Partial Choice Functions and Their Application to Belief Revision.
}

Ma, J., Liu, W., \& Dubois, D. (2015). Rational Partial Choice Functions and Their Application to Belief Revision. In Knowledge Science, Engineering and Management - Proceedings of 8th International Conference, KSEM 2015, (pp. 128-140). (Lecture Notes in Artificial Intelligence; Vol. 9403). Springer. https://doi.org/10.1007/978-3319-25159-2_11

Published in:

Knowledge Science, Engineering and Management - Proceedings of 8th International Conference, KSEM 2015,

Document Version:

Peer reviewed version

Queen's University Belfast - Research Portal:

Link to publication record in Queen's University Belfast Research Portal

Publisher rights

(C) 2015 Springer International Publishing AG

The final publication is available at Springer via http://link.springer.com/chapter/10.1007\%2F978-3-319-25159-2_11

\section{General rights}

Copyright for the publications made accessible via the Queen's University Belfast Research Portal is retained by the author(s) and / or other copyright owners and it is a condition of accessing these publications that users recognise and abide by the legal requirements associated with these rights.

Take down policy

The Research Portal is Queen's institutional repository that provides access to Queen's research output. Every effort has been made to ensure that content in the Research Portal does not infringe any person's rights, or applicable UK laws. If you discover content in the Research Portal that you believe breaches copyright or violates any law, please contact openaccess@qub.ac.uk. 


\title{
Rational Partial Choice Functions and Their Application to Belief Revision
}

\author{
Jianbing $\mathrm{Ma}^{1}$, Weiru $\mathrm{Liu}^{2}$ and Didier Dubois ${ }^{2,3}$ \\ ${ }^{1}$ School of Design, Engineering and Computing, \\ Bournemouth University, Bournemouth, BH12 5BB, UK, \\ ${ }^{2}$ School of Electronics, Electrical Engineering and Computer Science, \\ Queen's University Belfast, Belfast BT7 1NN, UK \\ ${ }^{3}$ IRIT, University Paul Sabatier, Toulouse Cedex 9, France \\ jma@bournemouth.ac.uk, w.liu@qub.ac.uk, dubois@irit.fr
}

\begin{abstract}
Necessary and sufficient conditions for choice functions to be rational have been intensively studied in the past. However, in these attempts, a choice function is completely specified. That is, given any subset of options, called an issue, the best option over that issue is always known, whilst in real-world scenarios, it is very often that only a few choices are known instead of all. In this paper, we study partial choice functions and investigate necessary and sufficient rationality conditions for situations where only a few choices are known. We prove that our necessary and sufficient condition for partial choice functions boils down to the necessary and sufficient conditions for complete choice functions proposed in the literature. Choice functions have been instrumental in belief revision theory. That is, in most approaches to belief revision, the problem studied can simply be described as the choice of possible worlds compatible with the input information, given an agent's prior belief state. The main effort has been to devise strategies in order to infer the agents revised belief state. Our study considers the converse problem: given a collection of input information items and their corresponding revision results (as provided by an agent), does there exist a rational revision operation used by the agent and a consistent belief state that may explain the observed results?
\end{abstract}

\section{Introduction}

Choice functions are commonly used in economic and social sciences to describe the process following which, faced with a ranking problem, an individual makes choices by selecting a few outcomes in each subset of possible outcomes (such a subset is called an issue); the selected outcomes are those to which no other outcomes can be strictly more preferred according to its preference. These observable choices testify of a non-directly observable preference relation. There has been a considerable amount of study about the definition of optimal choices under different types of preference relations (e.g., [2]). Equally interesting is the converse problem described as: given a choice function, that is, an abstract mapping that selects some desirable outcomes for each issue [20], does there exist an underlying rational preference relation from which this choice function can be derived? Several solutions to this problem exist for a long time, for instance [9, 
20,23]. However, in these attempts, the choice functions are full-fledged mappings that provide every issue with a set of mapped outcomes. In real-world scenarios, it is very often that choices of only a few issues are known, instead of choices over all the issues. Actually it is natural that given the whole set of issues, only a subset of them may have been exposed to the agent and hence the agent can only provide a limited response to the issues. Therefore, it is interesting to study conditions ensuring the existence of a rational preference that can reflect these choices. In this paper, we define the concept of partial choice functions and investigate their necessary and sufficient conditions for scenarios where only a few choices are known. We also examine the relationships between partial and (complete) choice functions and between their necessary and sufficient conditions.

Interestingly, our study and investigation result can be applied to study the converse belief revision problem. Belief revision $[1,16,10,13]$ performs belief change on an agent's beliefs when new evidence is received. In classical belief revision, it is a default assumption that there is a clear and consistent belief structure (e.g., a total preorder over possible worlds, the most plausible possible worlds of which generate the belief set) embedded in an agent's mind, based on which this agent performs revision upon receiving new information. Here, we consider the converse of this problem. That is, if there are several revision scenarios, each of which consisting of a piece of new information and its corresponding revision result (represented as pairs $\left(\mu_{i}, \phi_{i}\right)$ subsequently), then does there exist a rational belief structure from which these revision scenarios proceed. In other words, for each input $\mu_{i}$, does the agent exhibit the existence of a belief structure where $\phi_{i}$ is exactly the revision result based on $\mu_{i}$ in scenario $\left(\mu_{i}, \phi_{i}\right)$ ? Figures 1 and 2 provide intuitive illustration of the difference between classic belief revision and its converse problem. That is, in classic belief revision, given an agent's a priori belief state and a newly received piece of information, appropriate revision strategies (operators) are designed to revise the agent's belief set and derive a new one. Whilst the converse problem is: given a set of revision scenarios (pairs of evidence and revision result), does the agent exhibit the existence of a consistent prior epistemic state justifying these revision results? Here by epistemic state, we mean the ranking of possible worlds, hence the corresponding prior belief set.

Belief revision ${ }^{1}$ always respects the Success postulate such that the revision result $\left(\phi_{i}\right)$ should respect the new information $\left(\mu_{i}\right)$. Semantically, the models of the revision result should be a subset of the models of the formula representing the new information (i.e., $\operatorname{Mod}\left(\phi_{i}\right) \subseteq \operatorname{Mod}\left(\mu_{i}\right)$ ). Therefore, the converse belief revision problem reduces to what we have discussed above on inducing a preference relation for choices. Actually, belief revision theory has borrowed material from choice function theory via axioms 7 and 8 of the AGM framework. This does not come as a surprise. An extensive discussion of the relationships between choice function axioms and properties of non-monotonic consequences, a topic closely related to belief revision, can be found in [18, 17, 22]. These papers also include an extensive bibliography on that topic. The last two AGM axioms are in fact not characteristic of the revision problem and one may argue that, by means of the two axioms, the AGM approach chooses as much an uncertainty framework that is not probabilistic by means of such two axioms as it provides core axioms for belief revision (the first six).

\footnotetext{
${ }^{1}$ Here we do not consider non-prioritized belief revision which can be seen as belief merging.
} 


\section{$\Phi \circ \mu \quad ?$}

Fig. 1. Classic Belief Revision

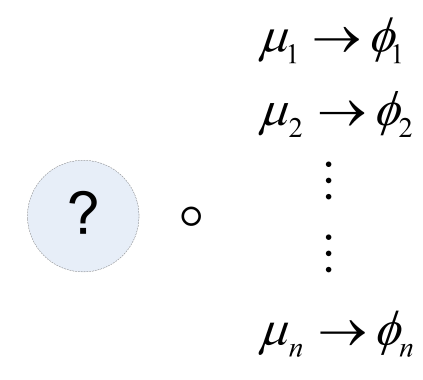

Fig. 2. Reversed Belief Revision

Note that the converse belief revision problem discussed here is different from the converse problem of iterated belief revision addressed in [8] and the converse belief revision in multi-agent systems [5]. Here, the multiple revision scenarios do not correspond to a sequence of revision actions, where the iteration may cause successive changes of an agent's belief structure. Instead, here we assume a given agent's belief structure, to which every new piece of information is applied separately so as to derive a different new belief state. This is in line with the view of belief revision as defeasible inference discussed in $[12,14]$, one of the three kinds of belief revision perspectives.

Below, we first provide some preliminaries on propositional logics and choice functions in Section 2. In Section 3, we define the notion of partial choice function and propose a necessary and sufficient condition for a partial choice function to be rational. We then compare our solution with related works in Section 4, showing that our condition can recover the conditions proposed in the literature. In Sections 5, we show how this condition can be used to solve the converse belief revision problem. Finally, in Section 6, we conclude the paper.

\section{Preliminaries}

Logic: We consider a propositional language $\mathcal{L}$ defined in a usual sense. We use $\operatorname{Mod}(\phi)$ to denote the set of models for $\phi$. We write $\phi \models \mu$ iff $\operatorname{Mod}(\phi) \subseteq \operatorname{Mod}(\mu)$. For a set $A$, $\operatorname{form}(A)$ denotes a formula whose set of models is $A$. That is, $\operatorname{Mod}(\operatorname{form}(A))=A$.

Choice Functions: Let $X$ denote the finite set of all conceivable outcomes, and $\mathcal{P}(X)$ represent the family of all non-empty subsets of $X$. Each $A \in \mathcal{P}(X)$ is called an issue (or agenda) and let $R$ denote a complete and reflexive binary relation defined on $X . R$ is a total pre-order if it is transitive, i.e., $x R y, y R z$ implies $x R z$ for any $x, y, z \in X$. 
Let $M(A, R)$ denote the set of maximal elements (choices) in $A$ such that:

$$
M(A, R)=\{x \mid x \in A \text { and } x R y, \forall y \in A\} .
$$

A choice function is a mapping $\mathcal{F}: \mathcal{P}(X) \rightarrow \mathcal{P}(X)$ satisfying: $\forall A \in \mathcal{P}(X), \mathcal{F}(A) \subseteq$ $A$. A choice function $\mathcal{F}$ is said to be rational, if there is a total preorder $R$ such that for all $A \in \mathcal{P}(X), \mathcal{F}(A)=M(A, R)$.

Elements $x$ and $y$ are called indifferent in terms of $R$, denoted $x \sim_{R} y$, if both $x R y$ and $y R x$ hold.

\section{Rational Partial Choice Functions}

If $\mathcal{F}$ is a mapping from $\mathcal{P}(X) \rightarrow \mathcal{P}(X)$, then for any issue $A \subseteq X$, we know the choice $\mathcal{F}(A)$ over $A$. But in real-world applications, usually we only know choices over a few issues. For instance, if we view a country as the set of its cities, then for a familiar country, we can tell our choices on preferred cities, while for unfamiliar countries, we cannot. Formally, we define the notion of a partial choice function.

Definition 1 Let $\Omega$ be a subset of $\mathcal{P}(X)$, a partial choice function $\mathcal{F}$ over $\Omega$ is a mapping: $\Omega \rightarrow \mathcal{P}(X)$ satisfying: $\forall A \in \Omega, \mathcal{F}(A) \subseteq A$.

Here $\Omega$ denotes the set of issues whose maximal elements are known. Note that in [6], although it is not explicitly defined, the choice frame proposed does contain a partial choice function.

Let $\bigcup \Omega=\bigcup_{A \in \Omega} A$ denote the union of all sets in $\Omega$. Similarly, we denote a partial choice function $\mathcal{F}$ over $\Omega$ is rational ${ }^{2}$ if there is a total pre-order $R$ over $\bigcup \Omega$ such that for all $A \in \Omega, \mathcal{F}(A)=M(A, R)$. Obviously, if $\Omega=\mathcal{P}(X)$, then our definition reduces to the standard definition.

Note that a total pre-order $R$ over $\bigcup \Omega$ can be easily extended to $X$ such that: for any $w, w^{\prime} \notin \bigcup \Omega, w \sim_{R} w^{\prime}$; and for $w \in \bigcup \Omega, w^{\prime} \notin \bigcup \Omega, w R w^{\prime}$ but not $w^{\prime} R w$ (this is a matter of convention, considering that options outside $\bigcup \Omega$ are less relevant here). With this extension, $A \in \Omega, \mathcal{F}(A)=M(A, R)$ still holds for any $A \in \Omega$.

Now we provide a necessary and sufficient condition ensuring the rationality of a partial choice function: Let $\left(A_{1}, \cdots, A_{i}, \cdots, A_{k}\right)$ be a sequence of issues and the circular permutation $\sigma(i)=i+1, i=1, \ldots, k-1$ and $\sigma(k)=1$.

Circular Consistency

$$
\begin{gathered}
\text { If } A_{\sigma(i)} \cap \mathcal{F}\left(A_{i}\right) \neq \emptyset, 1 \leq i \leq k, \\
\text { then } A_{\sigma(i)} \cap \mathcal{F}\left(A_{i}\right)=A_{i} \cap \mathcal{F}\left(A_{\sigma(i)}\right), 1 \leq i \leq k .
\end{gathered}
$$

This Circular Consistency condition shows that if an issue and the outcomes of the next issue are correlated circularly for $k$ issues, then this correlation establishes equivalent relations between issues and outcomes circularly.

We have the following result on two successive issues.

\footnotetext{
${ }^{2}$ An alternative definition would be to say a partial choice function $\mathcal{F}$ is rational if and only if it can be extended to a rational choice function over $\mathcal{P}(X)$ (i.e., there is a rational choice function over $\mathcal{P}(X)$ that agrees with $\mathcal{F}$ on $\Omega$ ). These two definitions are equivalent.
} 
Lemma 1 Let $\Omega=\left\{A, A^{\prime}\right\}$, if the Circular Consistency condition holds, and $A^{\prime} \cap$ $\mathcal{F}(A)=A \cap \mathcal{F}\left(A^{\prime}\right)$, then $A^{\prime} \cap \mathcal{F}(A)=\mathcal{F}(A) \cap \mathcal{F}\left(A^{\prime}\right)$.

Fig. 3 illustrates Lemma 1. The two circles represent $A_{i}$ and $A_{i+1}$, respectively. The intersections of the rectangle with the two circles represent $\mathcal{F}\left(A_{i}\right)$ and $\mathcal{F}\left(A_{i+1}\right)$, respectively, and the crossed section represents $\mathcal{F}\left(A_{i}\right) \cap \mathcal{F}\left(A_{i+1}\right)$. Now we give the

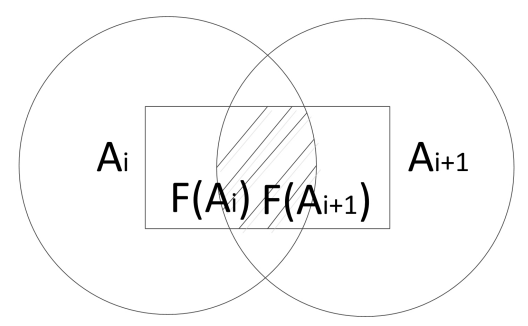

Fig. 3. Illustration of the two issue case

following representation theorem which shows that the above condition is necessary and sufficient to ensure the partial choice function being rational.

Theorem 1 Let $\mathcal{F}$ be a partial choice function over $\Omega$, then $\mathcal{F}$ is rational if and only if $\mathcal{F}$ satisfies the Circular Consistency condition.

Proof: The only if part is simple and we omit it here due to lack of space.

For the if part, assume that $\mathcal{F}$ satisfies the Circular Consistency condition.

We use graph theory to prove the existence of a total pre-order $R$. We view each issue $A \in \Omega$ as a point. If $\mathcal{F}\left(A_{i}\right) \cap \mathcal{F}\left(A_{j}\right) \neq \emptyset$, then we connect points $A_{i}$ and $A_{j}$ with an edge (no direction). Let $G$ denote the formed graph. Graph $G$ has several $(\leq n)$ connected components ${ }^{3}$, each of which contains a set of points (issues).

A simple fact that should be mentioned here is that for any two points $A_{i}, A_{j}$ in the same connected component, we could find a set of points $\left\{A_{t_{1}}, \cdots, A_{t_{m}}\right\}$ such that $A_{t_{1}}=A_{i}, A_{t_{m}}=A_{j}$ and for any $t_{s}, t_{s+1}, \mathcal{F}\left(A_{t_{s}}\right) \cap \mathcal{F}\left(A_{t_{s+1}}\right) \neq \emptyset$. That is, there is a path between points $A_{i}$ and $A_{j}$, which is ensured by the connectiveness of the connected component.

Now we view each connected component as a node. For two points $A_{i}, A_{j}$ in different nodes $X, Y$, respectively, if $\mathcal{F}\left(A_{i}\right) \cap A_{j} \neq \emptyset$, then we create a directed edge from

\footnotetext{
${ }^{3}$ In graph theory [4], a connected component of an undirected graph is a subgraph in which any two vertices are connected to each other by paths, and which (the subgraph) is connected to no additional vertices. A directed connected component of a directed graph is a subgraph in which any two vertices are connected to each other by a direct path, and which is connected to no additional vertices.

Graph $G$ has $n$ connected components only when all $A_{i}$ s are pairwise disjoint. In all other cases, $G$ has less than $n$ connected components. Here notice that the connected components, when viewed as a set of $A_{i} \mathrm{~s}$ (its points), are a partition of $\Omega$.
} 
$X$ to $Y$. If there are multiple directed edges from $X$ to $Y$, we compress them as one edge. This forms a new graph $G^{\prime}$.

Here we should point out an important fact: $G^{\prime}$ has no directed cycles. Otherwise suppose $G^{\prime}$ has a cycle $X_{1} \rightarrow X_{2} \rightarrow \cdots \rightarrow X_{k} \rightarrow X_{1}$. Without loss of generality, let points $A_{i_{1}}, A_{i_{2}}$ be contained in $X_{i}(1 \leq i \leq k)$ and $\mathcal{F}\left(A_{(i-1)_{2}} \cap A_{i_{1}} \neq \emptyset\right.$, $\mathcal{F}\left(A_{i_{2}}\right) \cap A_{(i+1)_{1}} \neq \emptyset$ hold (when $i=k, i+1$ is changed to 1 ; when $i=1$, $i-1$ is changed to $k$ ). As mentioned earlier, points $i_{1}$ and $i_{2}$ in connected component $X_{i}$ can also be connected by a path $\left\{A_{t_{1}}=A_{i_{1}}, \cdots, A_{t_{m_{i}}}=A_{i_{2}}\right\}$ such that $\mathcal{F}\left(A_{t_{s}}\right) \cap A_{t_{s+1}} \neq \emptyset$ (Actually it is $\mathcal{F}\left(A_{t_{s}}\right) \cap \mathcal{F}\left(A_{t_{s+1}}\right) \neq \emptyset$. However, clearly $\mathcal{F}\left(A_{t_{s}}\right) \cap \mathcal{F}\left(A_{t_{s+1}}\right) \neq \emptyset$ implies $\left.\mathcal{F}\left(A_{t_{s}}\right) \cap A_{t_{s+1}} \neq \emptyset\right), 1 \leq s<m_{i}$. Hence finally we get a cycle $\left\{A_{i_{1}}, \cdots, A_{i_{2}}, A_{(i+1)_{1}}, \cdots, A_{(i+1)_{2}}, \cdots, A_{(i-1)_{2}}, A_{i_{1}}\right\}$. For any two adjacent points $A_{l}, A_{l^{\prime}}$ in that cycle, we always have $\mathcal{F}\left(A_{l}\right) \cap A_{l^{\prime}} \neq \emptyset$. By the Circular Consistency condition and Lemma 1 , we get $\mathcal{F}\left(A_{(i-1)_{2}}\right) \cap \mathcal{F}\left(A_{i_{1}}\right)=\mathcal{F}\left(A_{(i-1)_{2}}\right) \cap A_{i_{1}} \neq \emptyset$. That is, points $A_{(i-1)_{2}}$ and $A_{i_{1}}$ should be in the same node, which creates a contradiction since they are in different nodes $X_{i-1}$ and $X_{i}$, respectively.

Since $G^{\prime}$ has no directed cycles, each of its directed connected component is a connected directed acyclic graph (DAG). For each of such DAG $H$ with nodes $Y_{1}, \cdots, Y_{k} \in$ $H$, we establish a mapping $f_{H}: H \rightarrow N$ associating each node with a natural number. To do this, first we associate each node with a number indicating its maximal level.

- Any node with 0 in-degree 4 is assigned level 0.

- Iteratively, a node is assigned with level $k$ if each of its parent nodes is assigned with a level value such that the maximal value among these is $k-1^{5}$.

With the concept of level of node, we define $f_{H}$ as follows.

- Any node with 0 in-degree (level 0 ) is assigned 1.

- Iteratively (by increasing the level value by 1 each time), for any node $Z$ with $s$ in-degree and hence has $s$ directed in-edges, $Z_{1} \rightarrow Z, \cdots, Z_{s} \rightarrow Z, f_{H}(Z)=$ $\sum_{i=1}^{s} f_{H}\left(Z_{i}\right)+s$.

This construction ensures that if a directed edge $Z^{\prime} \rightarrow Z$ exists, then $f_{H}\left(Z^{\prime}\right)<f_{H}(Z)$.

Now we establish a mapping $f: G \rightarrow N$ such that for any point $A_{i}$ in tree $H$ (in fact in a node $Y_{t}$ of $\left.H\right), f\left(A_{i}\right)=f_{H}\left(Y_{t}\right)$. Obviously we can see that any two points in the same sub-graph of $G$ (hence in the same node) have the same $f$ value.

Finally we establish a mapping $g: \bigcup \Omega \rightarrow N \cup\{0\}$ as follows.

If $w \in \mathcal{F}\left(A_{i}\right)$, then $g(w)=f\left(A_{i}\right)$. If $w \notin \bigcup_{A_{i} \in \Omega} \mathcal{F}\left(A_{i}\right)$, then $g(w)=0$.

Here we need to clarify that $g$ is a valid mapping. That is, we must show if $w \in$ $\mathcal{F}\left(A_{i}\right) \cap \mathcal{F}\left(A_{j}\right)$, then $f\left(A_{i}\right)=f\left(A_{j}\right)$. In fact, if $\mathcal{F}\left(A_{i}\right) \cap \mathcal{F}\left(A_{j}\right) \neq \emptyset$, then points $A_{i}$ and $A_{j}$ are in the same connected component of $G$, and hence $f\left(A_{i}\right)=f\left(A_{j}\right)$.

We define $R$ as: $w R w^{\prime}$ if and only if $g(w) \geq g\left(w^{\prime}\right)$. It remains to show $\forall i, \mathcal{F}\left(A_{i}\right)=$ $M\left(A_{i}, R\right)$.

By the definition of $g$, we know that if $w, w^{\prime} \in \mathcal{F}\left(A_{i}\right)$, then $g(w)=g\left(w^{\prime}\right)$. Now it is sufficient to show that if $w \in \mathcal{F}\left(A_{i}\right)$ and $w^{\prime} \in A_{i} \backslash \mathcal{F}\left(A_{i}\right)$, then $g(w)>g\left(w^{\prime}\right)$.

\footnotetext{
${ }^{4}$ The number of directed edges pointing to a node is called the in-degree of the node.

${ }^{5}$ Here we can prove that the level of a node is the maximal length of any path from some 0 in-degree node to this node. However, this result is not essential in our proof so we omit it here.
} 
If $w^{\prime} \notin \bigcup_{A_{i} \in \Omega} \mathcal{F}\left(A_{i}\right)$, then $g(w) \geq 1>0=g\left(w^{\prime}\right)$.

Else suppose $w^{\prime}$ is in some $\mathcal{F}\left(A_{j}\right)$ (hence $w^{\prime} \in A_{i} \cap \mathcal{F}\left(A_{j}\right)$ ), then $\mathcal{F}\left(A_{j}\right) \cap A_{i} \neq \emptyset$. If $\mathcal{F}\left(A_{j} \cap \mathcal{F}\left(A_{i}\right) \neq \emptyset\right.$, then according to Lemma 1, we have $\mathcal{F}\left(A_{j}\right) \cap \mathcal{F}\left(A_{i}\right)=\mathcal{F}\left(A_{j}\right) \cap$ $A_{i}$, hence $w^{\prime} \in \mathcal{F}\left(A_{i}\right) \cap \mathcal{F}\left(A_{j}\right)$ which contradicts with $w^{\prime} \notin \mathcal{F}\left(A_{i}\right)$.

Therefore, we should have $\mathcal{F}\left(A_{j}\right) \cap \mathcal{F}\left(A_{i}\right)=\emptyset$, which implies that points $A_{j}$ and $A_{i}$ are not in the same connected component of $G$, otherwise $\mathcal{F}\left(A_{j}\right) \cap \mathcal{F}\left(A_{i}\right)=\mathcal{F}\left(A_{j}\right) \cap$ $A_{i} \neq \emptyset$. Now we can conclude that in graph $G^{\prime}$, there is a directed edge from the node containing point $A_{j}$ to the node containing point $A_{i}$. Therefore, by the definition of $f_{H}$ and $f$, we know $f\left(A_{i}\right)>f\left(A_{j}\right)$. Hence $g(w)=f\left(A_{i}\right)>f\left(A_{j}\right)=g\left(w^{\prime}\right)$. This completes the proof.

Note that this proof also shows how to construct such a pre-order $R$ by which $\mathcal{F}$ is rationalized.

Fig. 4 illustrates the above theorem where each solid circle represents an $A_{i}$. The intersection of a solid circle (representing $A_{i}$ ) with the dashed red ring in the center represents $\mathcal{F}\left(A_{i}\right)$. Note that Fig. 4 just shows a sequence of issues that satisfy the

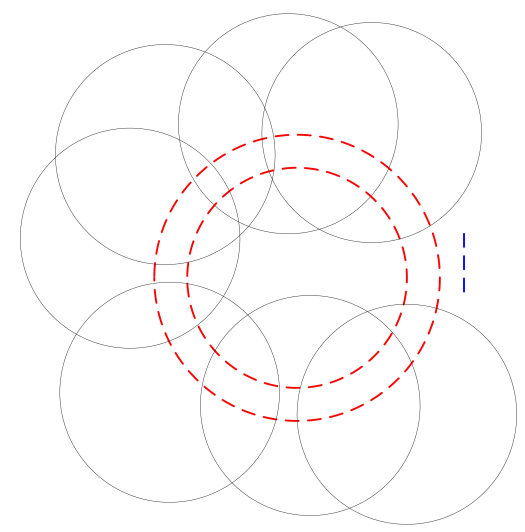

Fig. 4. Illustration of the $\mathrm{k}$ Issue Case

Circular Consistency condition. However, there could be multiple sequences (not necessarily disjoint), each of which satisfies the Circular Consistency condition. So Fig. 4 is not necessarily a complete view for all sets $A_{1}, \cdots, A_{n}$.

From Fig. 4, we can also see that $\forall x \in \mathcal{F}\left(A_{i}\right), y \in \mathcal{F}\left(A_{j}\right), 1 \leq i, j \leq k$, we have $x \sim_{R} y$ (as elements in the dashed red ring have the same plausibility). Note that for $z \in \mathcal{F}\left(A_{m}\right), m>k$, we do not necessarily have $x \sim_{R} z$.

\section{Related Work}

Classical works proved that a choice function $\mathcal{F}$ is rational if and only if it satisfies the following condition [20,23]:

Sen for all $B \in \mathcal{P}(X)$ and all $a \in B, a \in \mathcal{F}(B)$ if and only if $\forall b \in B, a \in \mathcal{F}(\{a, b\})$. 
As a corollary, when a partial choice function turns into a choice function, our Circular Consistency condition implies condition Sen.

Corollary 1 Let $\mathcal{F}$ be a choice function, then if it satisfies Circular Consistency, it satisfies Sen.

Also, in [9], Chernoff introduced a necessary condition for a choice function to be rational:

Chernoff for all $B, B^{\prime} \in \mathcal{P}(X)$, if $B \subseteq B^{\prime}$, then $\mathcal{F}\left(B^{\prime}\right) \cap B \subseteq \mathcal{F}(B)$.

Again, when a partial choice function turns into a choice function, our Circular Consistency condition also induces condition Chernoff.

Corollary 2 Let $\mathcal{F}$ be a choice function, then if it satisfies Circular Consistency, it satisfies Chernoff.

A lot of choice function conditions are reviewed in $[18,17,22]$ relating choice function theory to AGM theory and nonmonotonic reasoning. It can be shown that most of these conditions are derivable from our Circular Consistency condition. Since most of these conditions are straightforward to understand and also due to space limitation, here we omit most of them and only present the well-known Chernoff and Sen conditions. Interested readers can refer to [18] for details.

Now we first explore relationships between our approach to the well-known AGM framework. Let $K$ be a consistent belief set representing the agent's initial beliefs. $K$ is represented as a set of formulas. Let $B_{K}$ be a mapping from $K$ to any subset of formulas. The AGM axioms are listed as follows:

AGM1 $B_{K}(\phi)=\left[B_{K}(\phi)\right]^{P L}$ where for any set of formulas $S,[S]^{P L}$ is defined as the PL-deductive closure of $S$ in the usual way.

$\operatorname{AGM2} \phi \in B_{K}(\phi)$.

AGM3 $B_{K}(\phi) \subseteq[K \cup\{\phi\}]^{P L}$.

AGM4 If $\neg \phi \notin K$, then $[K \cup\{\phi\}]^{P L} \subseteq B_{K}(\phi)$.

AGM5 $B_{K}(\phi)=\Phi_{0}$ if and only if $\phi$ is a contradictory, where $\Phi_{0}$ is the set of formulas of the propositional language.

AGM6 If $\phi \leftrightarrow \psi$ is a tautology, then $B_{K}(\phi)=B_{K}(\psi)$.

AGM7 $B_{K}(\phi \wedge \psi) \subseteq\left[B_{K}(\phi) \cup\{\phi\}\right]^{P L}$.

AGM8 If $\neg \psi \notin B_{K}(\phi)$, then $\left[B_{K}(\phi) \cup\{\phi\}\right]^{P L} \subseteq B_{K}(\phi \wedge \psi)$.

Note that the Chernoff condition is very similar to the above AGM7 and AGM8 axioms ${ }^{6}$. It is easy to see that $B_{K}$ is a choice function at the semantic level. That is, $\operatorname{Mod}\left(B_{K}(\phi)\right) \subseteq \operatorname{Mod}(\phi)$ for any $\phi$. For simplicity, by abuse of notation, we write $B_{K}(\operatorname{Mod}(\phi)) \subseteq \operatorname{Mod}(\phi)$ (here notation $B_{K}$ is taken as a mapping from a set of models to another set of models). Now, let $B=\operatorname{Mod}(\psi \wedge \phi), B^{\prime}=\operatorname{Mod}(\phi)$ and $F=B_{K}$,

\footnotetext{
${ }^{6}$ The similarity between Chernoff and AGM7, AGM8 axioms can be shown more clearly if we define $B_{K}^{\prime}(\phi)=\bigwedge_{\psi \in B_{K}(\phi)} \psi$, and hence AGM7 and AGM8 axioms are translated as:

AGM7' $B_{K}^{\prime}(\phi) \wedge \psi \models B_{K}^{\prime}(\psi \wedge \phi)$.

AGM8' If $B_{K}^{\prime}(\phi) \not \models \neg \psi$, then $B_{K}^{\prime}(\psi \wedge \phi) \models B_{K}^{\prime}(\phi) \wedge \psi$.
} 
then $B \subseteq B^{\prime}$, and hence we obtain $B_{K}\left(B^{\prime}\right) \cap B \subseteq B_{K}(B)$ which is equivalent to $B_{K}(\phi) \wedge \psi \models B_{K}(\psi \wedge \phi)$.

A general condition called Arrow's condition [2] that generalizes both Sen and Chernoff says: if $B \subseteq B^{\prime}$, and $\mathcal{F}\left(B^{\prime}\right) \cap B \neq \emptyset$, then $\mathcal{F}(B)=\mathcal{F}\left(B^{\prime}\right) \cap B$. Indeed, if some of the best in $B^{\prime}$, belong to its subset $B$, none of the other elements in $B$ can be a best element in $B$ (they are dominated inside $B^{\prime}$ ). This is closely connected to Axiom 8 in the AGM framework.

In [6], the correspondence between AGM revision and rational choice functions is studied. It shows that a choice frame ${ }^{7}$ is rational if and only if it is AGM-consistent, where AGM-consistent is defined as: for every interpretation ${ }^{8}$ of the choice frame, the associated partial belief revision function can be extended to a full-domain belief revision function that satisfies the AGM-postulates.

Let $\operatorname{FORM}(A)=\{\phi: \operatorname{Mod}(\phi) \in A\}$ be a set of formulas whose set of models contain only elements of $A$. Note that $\operatorname{FORM}(A)$ is different from $\operatorname{form}(A)$. With this notation, for a partial choice function, the associated partial belief revision function is defined as follows.

Definition 2 Let $\mathcal{F}$ be a partial choice function such that $\mathcal{F}: \Omega \rightarrow \mathcal{P}(X)$, the associated belief revision function $B_{\mathcal{F}}$ is a mapping $B_{\mathcal{F}}: \operatorname{FORM}(\Omega) \rightarrow \operatorname{FORM}(\mathcal{P}(X))$ such that $B_{\mathcal{F}}(\phi)=\mathcal{F}(\operatorname{Mod}(\phi))$.

A full-domain belief revision function $B: F O R M(\mathcal{P}(X)) \rightarrow F O R M(\mathcal{P}(X))$ is called an extension of a partial belief revision function $B_{\mathcal{F}}$ if for any $\phi \in F O R M(\Omega)$, $B(\phi)=B_{\mathcal{F}}(\phi)$.

We can also define a partial choice function is AGM-consistent if its associated partial belief revision function can be extended to a full-domain belief revision function that satisfies the AGM-postulates.

Now we have the following corollary.

Corollary 3 Let $\mathcal{F}$ be a partial choice function, then it satisfies Circular Consistency if and only if it is AGM-consistent.

This Corollary suggests that there is an unearthed relationship between the Circular Consistency condition and the AGM postulates. We will leave this topic in future work.

Remarks: Although condition Chernoff looks similar to a Monotonicity property, Monotonicity does not hold for rational partial choice functions in general.

Mon If $A_{1} \subseteq A_{2}$, then $\mathcal{F}\left(A_{1}\right) \subseteq \mathcal{F}\left(A_{2}\right)$.

Example 1 Let $A_{1}=\left\{w_{1}, w_{2}\right\}, A_{2}=\left\{w_{1}, w_{2}, w_{3}\right\}, \mathcal{F}\left(A_{1}\right)=\left\{w_{1}, w_{2}\right\}$, and $\mathcal{F}\left(A_{2}\right)=$ $\left\{w_{3}\right\}$. We could see that $\mathcal{F}$ is a partial choice function over $\Omega=\left\{A_{1}, A_{2}\right\}$ and it is rational in terms of a pre-order $\preceq$ such that $w_{3} \prec w_{1} \simeq w_{2}$, i.e., we indeed have $\mathcal{F}\left(A_{1}\right)=M\left(A_{1}, \preceq\right)$ and $\mathcal{F}\left(A_{2}\right)=M\left(A_{2}, \preceq\right)$. But it does not satisfy the Monotonicity property. That is, we have $A_{1} \subset A_{2}$, but we do not have $\mathcal{F}\left(A_{1}\right) \subseteq \mathcal{F}\left(A_{2}\right)$.

\footnotetext{
${ }^{7}$ In [6], a choice frame consists of a set of alternatives $X$, a collection $\Omega$ of subsets of $X$, and a partial choice function $\mathcal{F}: \Omega \rightarrow \mathcal{P}(X)$.

${ }^{8}$ In [6], an interpretation of a choice frame is obtained by adding a valuation $V$ that assigns to every atom $p$ the subset of $X$ at which $p$ is true, and it is extended to formulas in the usual way. This is a standard definition of an interpretation.
} 


\section{Converse Belief Revision Problem}

As mentioned in Introduction, our result can be applied to the converse belief revision problem. Here we provide two necessary and sufficient conditions such that if a set of revision scenarios satisfy these two conditions, then there must be a consistent belief structure in the agent's mind, and vice versa.

To proceed, first, let us formalize the definition of a revision scenario.

Definition 3 (Revision scenario) A revision scenario, denoted as $r s=(\mu, \phi)$, is a pair of formulae $\mu$ and $\phi$, where $\mu$ represents a new piece of evidence and $\phi$ represents the revision result upon receiving $\mu$.

Below, we simply call a revision scenario a scenario.

Now we propose two conditions, i.e., Success and Circular Consistency conditions. Let $r s_{i}=\left(\mu_{i}, \phi_{i}\right), 1 \leq i \leq n$ be $n$ distinct revision scenarios.

Success For any $r s_{i}=\left(\mu_{i}, \phi_{i}\right), \phi_{i} \models \mu_{i}, 1 \leq i \leq n$.

Explanation: This is a variant of the well-known success postulate in the AGM framework. The revision result should respect the evidence.

Circular Consistency Let $\left\{\left(\mu^{1}, \phi^{1}\right), \cdots,\left(\mu^{k}, \phi^{k}\right)\right\}$ be any set of revision scenarios (i.e., a subset of the set of all $r s_{i}$ s) such that $k \leq n$, and let $\sigma$ be a circular permutation such that $\sigma(i)=i+1, i=1 \ldots k, \sigma(k)=1$. Then

$$
\begin{gathered}
\text { If } \mu^{\sigma(i)} \wedge \phi^{i} \not \models \perp, 1 \leq i \leq k, \\
\text { Then } \mu^{\sigma(i)} \wedge \phi^{i} \equiv \mu^{i} \wedge \phi^{\sigma(i)}, 1 \leq i \leq k
\end{gathered}
$$

As a corollary of Theorem 1, the following representation theorem shows that the above two conditions are necessary and sufficient to ensure that an agent has a consistent belief structure.

Corollary 4 Let $\mathcal{R S}$ be a set of scenarios, then there exists a belief structure, represented by a total pre-order $\preceq$ over $W$, s.t., $\forall r s=(\mu, \phi) \in \mathcal{R S}, \phi=\min (\operatorname{Mod}(\mu), \preceq)$ if and only if scenarios in $\mathcal{R S}$ satisfy Success and Circular Consistency.

Example 2 Suppose a hacking action was discovered originating from one of the PCs in an office. The log file shows that the action was conducted between $4 \mathrm{pm}$ to $5 \mathrm{pm}$. A suspect $Y$ declared that he left the office between 4pm and $5 \mathrm{pm}$ and he did not log on to any computer before he left. A witness suggested that $Y$ was seen doing something with that particular computer at that time. Now if suspect $Y$ explains:

Viewing web pages: $Y$ was viewing web pages, $O R$

Cleaning the monitor: $Y$ was cleaning the equipment.

Does $Y$ have a consistent belief structure with each one of these two explanations? The answer to the above question is important. Certainly, we cannot say $Y$ is innocent if $Y$ does have a consistent belief structure, as it only shows that $Y$ 's statements are coherent. However, $Y$ should be considered highly suspiciously if he fails to demonstrate consistency in its response to questions/evidence. 
Here suppose a system security engineer has two pieces of evidence: there was a hacking action during $4 \mathrm{pm}$ to $5 \mathrm{pm}$ and $Y$ was seen using the $\mathrm{PC}$ at the time. $Y$ 's reactions to the two pieces of evidence may allow the security engineer spotting an inconsistency between the statements, or equivalently, establishing whether $Y$ is applying a consistent belief structure when responding to the two inputs.

Let $\mu_{1}$ be 'a hacking action was discovered from $4 \mathrm{pm}$ to $5 \mathrm{pm}$ ', $\psi_{1}$ be ' $Y$ did not $\log$ on to any computer in the office'. Then $\phi_{1}=\mu_{1} \wedge \psi_{1}$ is $Y$ 's revision result upon receiving $\mu_{1}$, meaning that "although there was a hacking action, it is not me (i.e., $Y$ ) since I did not log on to any computer". Let $\mu_{2}$ be ' $Y$ was seen doing something with the $\mathrm{PC}$ at that time'. To consider possible revision results for the two situations described above, let $\phi_{2}$ be ' $Y$ was viewing web pages', and $\phi_{2}^{\prime}$ be ' $Y$ was cleaning the monitor'. Here we should note that $\phi_{1} \wedge \phi_{2} \equiv \perp$ since if $Y$ was viewing web pages, then $Y$ must have logged on to the $P C$, conflicting with $Y$ 's claim not logging on to any computers in the office. In this situation, as we only focus on two possibilities: ' $Y$ was viewing web pages' and ' $Y$ was cleaning the monitor', for simplicity we can assume that these two are exclusive and exhaustive. Hence it is easy to see that ' $Y$ was cleaning the monitor' indicates ' $Y$ did not $\log$ on to the $\mathrm{PC}$ ' and ' $Y$ was viewing web pages' indicates ' $Y$ had logged on to the PC', therefore we have $\phi_{2}=\mu_{2} \wedge \neg \psi_{1}$ and $\phi_{2}^{\prime}=\mu_{2} \wedge \psi_{1}$.

Now to justify whether $Y$ has a consistent belief structure, we check the two postulates.

Success Obviously we have $\phi_{1} \models \mu_{1}, \phi_{2} \models \mu_{2}$, and $\phi_{2}^{\prime} \models \mu_{2}$.

Circular Consistency For the two revision results $\phi_{2}$ and $\phi_{2}^{\prime}$,

- we have $\mu_{1} \wedge \phi_{2} \not \forall \perp$ and $\mu_{2} \wedge \phi_{1} \not \forall \perp$, but we do not have $\mu_{1} \wedge \phi_{2} \equiv \mu_{2} \wedge \phi_{1}$, - we have $\mu_{1} \wedge \phi_{2}^{\prime} \not \models \perp$ and $\mu_{2} \wedge \phi_{1} \not \models \perp$, and we have $\mu_{1} \wedge \phi_{2}^{\prime} \equiv \mu_{2} \wedge \phi_{1}$.

Therefore, by Corollary 4, we obtain the following conclusions:

- If the revision result by $Y$ is ' $Y$ was viewing web pages', then $Y$ does not have a consistent belief structure, and hence $Y$ is highly suspect.

- If the revision result by $Y$ (his explanation) is ' $Y$ was cleaning the monitor, then $Y$ has a consistent belief structure, and hence $Y$ might be innocent.

These conclusions are in line with our intuition. In the second case, a belief structure for $Y$ is: $\preceq:\left\{\mu_{1} \wedge \psi_{1} \prec \mu_{1} \wedge \neg \psi_{1} \simeq \mu_{2} \wedge \psi_{1} \prec \mu_{2} \wedge \neg \psi_{1}\right\}$. Here note that there are both models for $\psi_{1}$ and models for $\neg \psi_{1}$ appearing in $Y$ 's belief structure. This is because it is an inferred belief structure from the current information. $Y$ 's actual belief structure could be more elaborated, but should be consistent with the inferred belief structure.

\section{Conclusion}

In this paper, we proved a necessary and sufficient condition, i.e., Circular Consistency, for a partial choice function to be rational. Also, we have demonstrated that this solution can be used to construct a consistent belief structure for an agent from a set of revision scenarios.In addition, by comparing with related works, we proved that when a partial 
choice function turns into a choice function, our Circular Consistency condition can recover the necessary and sufficient conditions proposed before.

In terms of belief revision, our study hinted why lying could be a hard thing to do: one always needs to keep a track of what had been said to maintain consistency between different scenarios. This commonsense is widely acknowledged, and it is again partially proved in this paper in the belief revision scenario. Our study also provided some insights about belief revision in general.

As future work, one attempt is to investigate whether there are simpler conditions that can be used to replace the Circular Consistency condition, especially on whether the Circular Consistency condition can be decomposed into some AGM-like or Sen, Chernoff-like conditions. In addition, there is a notion called sub-rationality introduced by Deb [11] such that a choice function $F$ is sub-rational if for some order $R$, $M(A, R) \subseteq \mathcal{F}(A)$ holds for all $A \in \mathcal{P}(X)$. It is interesting to study the necessary and sufficient conditions for sub-rational partial choice functions.

\section{References}

1. C. E. Alchourrón, P. Gärdenfors, and D. Makinson. On the logic of theory change: Partial meet functions for contraction and revision. J. Sym. Log., 50:510-530, 1985.

2. K. Arrow. Uncertainty and the welfare economics of medical care. American Economic Review, 53(5):941-973, 1963.

3. S. Benferhat, D. Dubois, H. Prade, and M. A. Williams. A general framework for revising belief bases using quatitative Jeffrey's rule. Funda. Inform., 99:1-22, 2010.

4. N. Biggs, E. Lloyd, and R. Wilson. Graph Theory. Oxford University Press, 1986.

5. J. Biskup and C. Tadros. Revising belief without revealing secrets. In In Procs. of FoIKS, pages 51-70, 2012.

6. G. Bonanno. Rational choice and AGM belief revision. Artificial Intelligence, 173(1213):1194-1203, 2009.

7. R. Booth and T. Meyer. Admissible and restrained revision. JAIR, 26:127-151, 2006.

8. R. Booth and A. Nittka. Reconstructing an agent's epistemic state from observations. In Procs. of IJCAI, pages 394-399, 2005.

9. H. Chernoff. Rational selection of decision functions. Econometrica, 22(4):422-443, 1954.

10. A. Darwiche and J. Pearl. On the logic of iterated belief revision. Artificial Intelligence, 89:1-29, 1997.

11. R. Deb. Binariness and rational choice. Mathematical Social Sciences, 5:97-106, 1983.

12. J. Delgrande, D. Dubois, and J. Lang. Iterated revision as prioritized merging. In Proceedings of Knowledge Representation and Reasoning, (KR'06), pages 210-220, 2006.

13. J. Delgrande and Y. Jin. Parallel belief revision: Revising by sets of formulas. Artificial Intelligence, 176(1):2223-2245, 2012.

14. D. Dubois. Three scenarios for the revision of epistemic states. Journal of Logic and Compution, 18(5):721-738, 2008.

15. Y. Jin and M. Thielscher. Iterated belief revision, revised. Artificial Intelligence, 171:1-18, 2007.

16. H. Katsuno and A. O. Mendelzon. Propositional knowledge base revision and minimal change. Artificial Intelligence, 52:263-294, 1991.

17. D. Lehmann. Nonmonotonic logics and semantics. Journal of Logic and Computation, 11(2):229-256, 2001.

18. S. Lindström. A semantic approach to nonmonotonic reasoning: inference operations and choice. 1994. 
19. J. Ma, W. Liu, and S. Benferhat. A belief revision framework for revising epistemic states with partial epistemic states. In Procs. of AAAI, pages 333-338, 2010.

20. H. Moulin. Choice functions over a finite set: A summary. Social Choice and Welfare, 2(2):147-160, 1985

21. A. C. Nayak, M. Pagnucco, and P. Peppas. Dynamic belief revision operators. Artificial Intelligence, 146:193-228, 2003.

22. H. Rott. Change, choice and inference. Clarendon Press, Oxford, 2001.

23. A. K. Sen. Internal consistency of choice. Econometrica, 61(3):495-521, 1993. 